transport to the surface at that point, i.e.,

$$
k c=0.339 S_{c} \beta\left(\frac{L}{x}\right)^{\frac{1}{3}}(a-c),
$$

where $c$ is the unknown surface concentration. The basic assumption in this formulation is that the rate of mass transport is presumably uninfluenced by the surface reaction. Solving for $c$ one finds with Eq. (47) that

$$
c(z)=\frac{a}{z+1}
$$

This is shown for comparison in Fig. 2. This result does not agree with Eq. (54) and is not correct although the numerical differences are relatively moderate in this particular example.

On the other hand, the validity of our results must also be checked from the point of view of the boundary layer assumptions. Chapman and Rubesin have shown ${ }^{8}$ that if a property variation occurs on a surface, the gradient in the $x$ direction must on account of these assumptions be limited to

$$
\left[\frac{d\left(\frac{c_{0}}{a}\right)}{d\left(\frac{x}{L}\right)}\right]_{y=0} \sim \frac{\left(1-\frac{c_{0}}{a}\right) L}{5\left(\frac{\nu x}{U_{\infty}}\right)^{\frac{1}{2}}}
$$

which is of the order of $(k L / D) S_{c}^{-\frac{1}{3}}$ at the leading edge. This is apparently not too serious a restriction in practice. However, sufficiently far downstream the solution is valid without further qualifications.

The analysis has been generalized to apply to chemical surface reactions in other types of boundary layer flows both laminar as well as turbulent. This has been considered, together with the nonisothermal aspects, by one of the authors in a paper to appear shortly. Another paper by the authors will deal with the application and the numerical solution of the equations derived above to some specific cases of interest in chemical engineering.

\title{
Ferrite Post in a Rectangular Wave Guide
}

\author{
P. S. Epstejn, Califormia Institute of Technology, Pasadena, California \\ AND
}

A. D. BERK, Hughes Aircraft Company, Culver City, California

(Received May 18, 1956)

\begin{abstract}
A thin circular ferrite post magnetized lengthwise is placed in a rectangular wave guide with its axis normal to the direction of propagation of the incident waves. The polarization is such that the electric vector is parallel to the post. The reflected and transmitted waves are calculated both with respect to their intensities and phases. The results are also applied to find the influence of a thin ferrite post upon the resonant frequency of a rectangular cavity.
\end{abstract}

\section{INTRODUCTION}

$\mathbf{M}$ ICROWAVE guides containing ferrite materials have been investigated by a number of authors. ${ }^{1-5}$ Most of these investigations were confined to those configurations where the distribution of ferrite material is uniform along the direction of propagation. However, ferrite obstacles in wave guides are also of interest. In this paper we shall study one of the simplest of wave-guide-obstacle configurations, namely, a circular cylindrical ferrite pencil (or post) placed parallel to one pair of the sides of a rectangular wave guide and magnetized axially by a constant magnetic field. The

${ }^{1}$ C. L. Hogan, Bell System Tech. J. 31, 1 (1952).

2 M. L. Kales, J. Appl. Phys. 24, 604 (1953).

${ }^{3}$ H. J. Gamo, J. Phys. Soc. Japan, 8, 176 (1953).

4 H. Suhl and L. R. Walker, Bell System Tech. J. 33, 519, 939, 1133 (1954).

${ }^{5}$ Lax, Button, and Roth, J. Appl. Phys. 25, 1413 (1954). polarization of the incident microwave is chosen so that the electric vector is parallel to the pencil axis.

The interest of this problem is not entirely theoretical. Its solutions will contribute to the detailed understanding of the new ferrite directional couplers developed at General Electric Company, ${ }^{6}$ and at Hughes Research Laboratories. ${ }^{7}$ Besides, Sec. 10 of this paper brings the discussion of a resonant cavity containing a ferrite post, which may be helpful in the accurate measurement of some of the ferrite constants.

Our results show that in the case of a ferrite post whose diameter is small compared with the distance to the nearest side wall only the first few terms of the

\footnotetext{
${ }^{6}$ R. W. Damon, J. Appl. Phys. 26, 1280 (1955).

${ }^{7}$ A. D. Berk and E. Strumwasser, Ferrite Directional Couplers paper presented at the Symposium on Microwave Properties and Applications of Ferrites, Harvard University, April 4, 1956. See Proc. Inst. Radio Engrs. 10 (1956).
} 

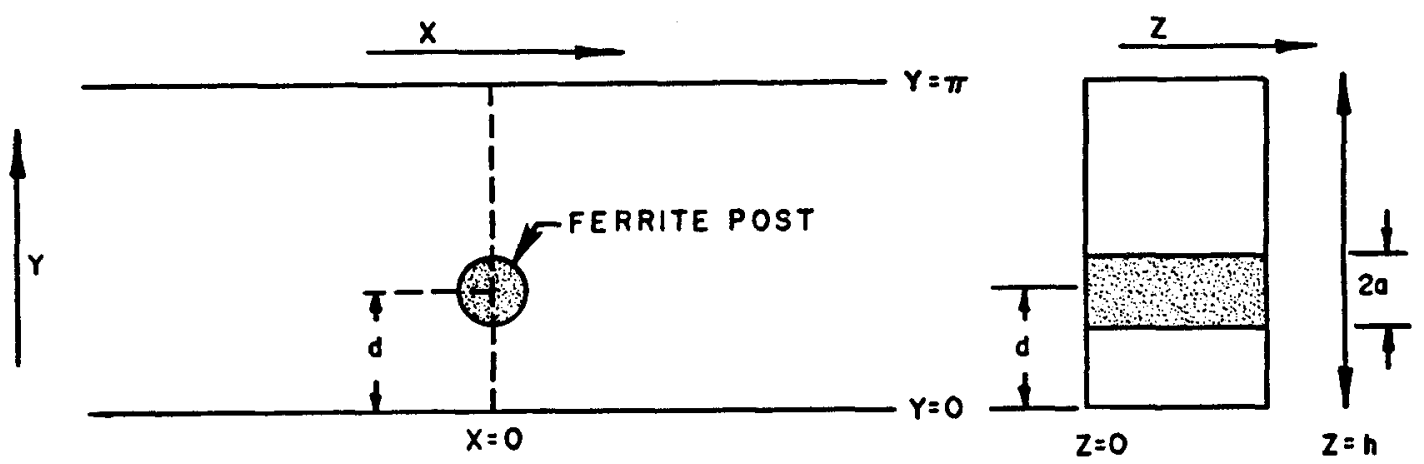

Fic. 1. Ferrite post in a rectangular wave guide.

series representing the reflection and transmission coefficients need be retained. For very thin posts the first terms of these series are entirely adequate. When specialized for the case of a nonmagnetic dielectric post, our results agree with those already given for the latter by Marcuvitz. ${ }^{8}$

\section{MATHEMATICAL FORMULATION OF THE PROBLEM}

Let us consider a rectangular wave guide extending without limit in the $x$ direction, having the width $l$ in the $y$ direction and the height $h$ in the $z$ direction. It is convenient to choose the units of length so that the width $l$ becomes numerically equal to $\pi(l=\pi)$, so that

$$
-\infty<x<+\infty, 0<y<\pi, \quad 0<z<h .
$$

Inserted into the wave guide is a ferrite post in the form of a circular cylinder magnetized lengthwise whose axis is parallel to $z$ and located at $x=0, y=d$ (see Fig. 1). We ask: what is the field within this system when the electric vector has the $z$ direction and is thus parallel to the axis of the post?

The boundary conditions at the walls of the wave guide are

$$
E_{t}=0,
$$

where the subscript $t$ refers to a tangential component. In view of our assumption we can let

$$
E_{z}=E, \quad E_{x}=E_{y}=0,
$$

for the whole interior of the wave guide.

Therefore, the condition (2.2) is automatically satisfied at the two walls $z=0, z=h$. We shall show that this makes it possible to treat the problem as two-dimensional inasmuch as all its characteristics become independent of the coordinate $z$, so that the electric field becomes

$$
E=E_{z}=E(x, y) \text {, }
$$

and is a function of the two variables $x, y$ only.

Indeed, in the empty space outside the post the function $E$ must satisfy the familiar form the wave equation

${ }^{8}$ N. Marcuvitz, Wave-Guide Handbook (Mass. Inst. Technol. Rad. Lab. Series, 1951), p. 260. takes when only periodic states of the field with the time factor $\exp (-i \omega t)$ are considered

$$
\left(\nabla^{2}+k_{0}^{2}\right) E=0, \quad k_{0}=\omega / c,
$$

where $k_{0}$ is the wave number in empty space, $\omega$ the angular frequency, and $c$ the velocity of light.

The magnetic field lies then in the $(x, y)$ plane being given by the two-dimensional tensor equation

$$
\mathbf{H}=-\left(i / k_{0}\right)\left(\begin{array}{rr}
0 & 1 \\
-1 & 0
\end{array}\right) \nabla_{p} E
$$

where $\nabla_{p}$ denotes the two-dimensional symbolic gradient $\partial / \partial x, \partial / \partial y$.

On the other hand, the ferrite material of the post is characterized by the scalar dielectric constant $\epsilon$ and by the tensor permeability

$$
(\mu)=\left(\begin{array}{ccc}
\mu_{1} & i_{\kappa} & 0 \\
-i_{\kappa} & \mu_{1} & 0 \\
0 & 0 & \mu_{3}
\end{array}\right),
$$

where the rows and columns refer to the directions $x, y$, $z$, respectively. It is well to mention here that the component $\mu_{3}$ does not come into play because the magnetic field is normal to the $z$ direction. The electric field within the ferrite is restricted to the component $E^{\prime}=E_{z}$ which is subject to the wave equation ${ }^{9}$

$$
\begin{gathered}
\left(\nabla_{p}^{2}+k^{2}\right) E^{\prime}=0, \quad k^{2}=\epsilon k_{0}^{2} / M, \\
M=\mu_{1}\left(\mu_{1}^{2}-\kappa^{2}\right)^{-1}, \quad K=-\kappa\left(\mu_{1}^{2}-\kappa^{2}\right)^{-1} .
\end{gathered}
$$

The magnetic field intensity lies again in the $(x, y)$ plane and is given by

$$
\mathbf{H}^{\prime}=\left(i / k_{0}\right)\left(\begin{array}{rr}
i K & -M \\
M & i K
\end{array}\right) \nabla_{p} E^{\prime} .
$$

The boundary conditions which the field has to satisfy are as follows: The Eqs. (2.2) require, in addition to $(2.3)$, the condition

$$
E=0, \quad \text { for } y=0, y=\pi .
$$

${ }^{9}$ Compare, for instance, P. S. Epstein, Revs. Modern Phys. 28, 7 (1956), Sec. 6, Eq. (6.5). 
Besides, at the surface of the post the tangential components of the electric and magnetic fields must change continuously:

$$
E=E^{\prime}, \quad H_{t}=H_{t}^{\prime} .
$$

We can give now a precise formulation of the problem we wish to treat. Let the field in the wave guide, in the absence of the ferrite post, be represented by the mode

$$
\begin{aligned}
& E_{n}=\sin n y \exp \left(i \gamma_{n} x\right), \\
& \gamma_{n}=\left(k_{0}{ }^{2}-n^{2}\right)^{\frac{1}{2}},
\end{aligned}
$$

where $n$ is an integral number. We call $E_{n}$ the incident wave and we ask: What change of field is produced by the insertion of the post? This change is expressed mathematically by the appearance of a perturbation field $E_{p}$ superposed upon the incident wave. Thus, the total field outside the post (denoted above by $E$ ) becomes $E=E_{n}+E_{p}$, while $E^{\prime}$ of the Eqs. (2.8), (2.10), (2.12) is the total field inside the post.

We shall be particularly interested in the perturbation field at considerable distances (positive or negative) from the post in the $x$ direction. Because of the mathematical difficulties of the problem, we shall have to be satisfied with finding the answer for comparatively thin posts; hence the method of investigation will be adapted to this case.

\section{METHOD OF INVESTIGATION}

Assuming that the radius $a$ of the post is small compared with the distance $d$ from its axis to the nearest side wall $(y=0)$ of the wave guide and compared with the wavelength $\lambda$ in free space, a method of treatment by successive approximations naturally presents itself. It is best to describe the consecutive steps in physical terms, as follows:

\section{(1) The Scattered Waves of the First Order}

$E_{s 1}, E_{s 1}{ }^{\prime}$ (outside and inside the post) are obtained by choosing $E_{n}+E_{\mathrm{s} 1}, E_{s 1}{ }^{\prime}$ so as to satisfy the conditions (2.12) at the surface of the post. In other words, these waves are calculated as if the incident wave (2.13) were falling on the post in an unlimited space without any side walls at $y=0$ and $y=\pi$.

\section{(2) The Mirrored Wave of the First Order, $E_{m 1}$}

The scattered wave $E_{s 1}$ does not satisfy the boundary conditions (2.11) at the side surfaces. To fulfill them we introduce an additional wave $E_{m 1}$ so that the Eqs. (2.11) are satisfied by the sum $E_{81}+E_{m 1}$.

\section{(3) The Scattered Waves of the Second Order, $E_{s 2}, E_{s 2^{\prime}}$}

The mirrored wave $E_{m 1}$ does not satisfy the boundary conditions at the surface of the post. Hence, additional waves must be introduced so that $E_{m 1}+E_{82}$ (outside) together with $E_{s 2}{ }^{\prime}$ (inside) satisfy the Eqs. (2.12). As compared with the scattered wave of the first order, $E_{s 1}$, the wave $E_{82}$ is considerably attenuated for two reasons. In the first place, because of the basic assumption of a thin post $(a<d)$ the intensity of the first mirrored wave, $E_{m 1}$, at the surface of the post is small in comparison with $E_{s 1}$ standing with it in the ratio $(a / d)$. In the second place, since the radius $a$ is also small compared with the wavelength in vacuo, every successive scattering from the thin post leads to a further attenuation. It is difficult to estimate this second effect accurately because of the presence of evanescent modes in $E_{s 1}$. We shall discuss it in Sec. 9 where a strong argument will be presented.

\section{(4) The Mirrored Wave of the First Order, $E_{m 2}$}

This stands in the same relation to $E_{s 2}$ as $E_{m 1}$ stands to $E_{s 1}$.

In this way it is conceptually possible to continue the process indefinitely and to go to scattered waves of higher and higher order. However, we shall restrict ourselves to the case when $a / d$ is sufficiently small for waves of the second order to be negligible, so that the operations listed under (1) and (2) are sufficient to furnish a solution of the problem of the desired accuracy. The calculation of the first order scattering does not offer any difficulties and will be carried out in Secs. 4 and 5 . Of the various ways of obtaining the mirrored wave of the first order, the method of the Green's function was found to be the simplest. Its application to our problem is contained in Secs. 6, 7,8.

It may be emphasized that the method described here is suitable only for sufficiently thin posts so that the scattered and the mirrored waves of the first order already give a sufficient approximation. Pushing it to approximations higher than the first would be not only difficult but perhaps even unfeasible as will appear in Sec. 9.

\section{SCATTERED WAVE OF THE FIRST ORDER}

It is convenient to write the incident wave $(2.13)$ in a slightly different form by introducing an auxiliary angle $\epsilon_{n}$ defined by the Eqs.

$$
\gamma_{n}=k_{0} \cos \epsilon_{n}, \quad n=k_{0} \sin \epsilon_{n}, \quad \operatorname{tg} \epsilon_{n}=n / \gamma_{n},
$$

whence

$$
E_{n}=\sin \left(k_{0} y \sin \epsilon_{n}\right) \exp i\left(k_{0} x \cos \epsilon_{n}\right) .
$$

For our applications it is necessary to represent this function in polar coordinates $r, \varphi$ of the $(x, y)$ plane referred to the axis of the post $(x=0, y=d)$ as their origin

so that

$$
x=r \cos \varphi, \quad y=d+r \sin \varphi,
$$

$$
E_{n}=\sin \left(n d+k_{0} r \sin \varphi \sin \epsilon_{n}\right) \exp i\left(k_{0} r \cos \varphi \cos \epsilon_{n}\right) .
$$


The expansion into a Fourier series is well known, namely,

$$
E_{n}=\sum_{\nu=0}^{\infty} J_{\nu}\left(k_{0} r\right)\left[p_{n \nu} \cos \nu \varphi+q_{n \nu} \sin \nu \varphi\right],
$$

with the abbreviations

$$
\left.\begin{array}{l}
p_{n 0}=\sin n d, \quad q_{n 0}=0, \\
p_{n \nu}=2 i^{\nu} \cos \nu \epsilon_{n} \sin n d, \\
q_{n \nu}=2 i^{\nu-1} \sin \nu \epsilon_{n} \cos n d, \quad \nu=1,2,3, \ldots
\end{array}\right\}
$$

According to Eq. (2.6) the $\varphi$ component of the magnetic field is

$$
\begin{aligned}
& H_{n \varphi}=\left(i / k_{0}\right) \frac{\partial E_{r}}{\partial r} \\
&=i \sum_{\nu=0}^{\infty} J_{\nu}{ }^{\prime}\left(k_{0} r\right)\left[p_{n \nu} \cos n \varphi+q_{n \nu} \sin n \varphi\right] .
\end{aligned}
$$

As we shall take into account the scattered wave of the first order only, we shall designate it by the single subscript $s$, omitting the second subscript 1 . The general expression of its electric field is

$$
E_{s}=\sum_{\nu=0}^{\infty} H_{\nu}^{(1)}\left(k_{0} r\right)\left[A_{\nu} \cos \nu \varphi+B_{\nu} \sin \nu \varphi\right]
$$

where $H_{v}{ }^{(1)}\left(k_{0} r\right)$ denotes the Hankel function of the first kind and $A_{\nu}, B_{\nu}$ are arbitrary coefficients. The $\varphi$ component of the magnetic field is then,

$$
H_{s \varphi}=i \sum_{\nu=0}^{\infty} H_{\nu}^{(1)^{\prime}}\left(k_{0} r\right)\left[A_{\nu} \cos \nu \varphi+B_{\nu} \sin \nu \varphi\right]
$$

The electric field inside the post satisfies Eq. (2.8) and must be written in the form

$$
E_{s}^{\prime}=\sum_{\nu=0}^{\infty} J_{\nu}(k r)\left[A_{\nu}^{\prime} \cos \nu \varphi+B_{\nu}^{\prime} \sin \nu \varphi\right]
$$

The magnetic field follows from the expression (2.10). The $\varphi$ component is thus

$$
\begin{aligned}
& H_{s \varphi}{ }^{\prime}=\left(i / k_{0}\right) \sum_{\nu=0}^{\infty}\left\{\left[A_{\nu}{ }^{\prime} M k J_{\nu}{ }^{\prime}(k r)\right.\right. \\
& \left.+B_{\nu}{ }^{\prime} i K(\nu / r) J_{\nu}(k r)\right] \cos \nu \varphi \\
& \left.+\left[-A_{\nu}{ }^{\prime} i K(\nu / r) J_{\nu}(k r)+B_{\nu}{ }^{\prime} M k J_{\nu}{ }^{\prime}(k r)\right] \sin \nu \varphi\right\}
\end{aligned}
$$

The conditions (2.12) at the surface of the ferrite post, $r=a$, take now the form

$$
E_{n}+E_{s}=E_{s}^{\prime}, \quad H_{n \varphi}+H_{s \varphi}=H_{s \varphi}^{\prime},
$$

and lead to the system of equations

$$
\begin{aligned}
p_{n \nu} J_{\nu}\left(k_{0} a\right)+A_{\nu} H_{\nu}{ }^{(1)}\left(k_{0} a\right) & =A_{\nu}{ }^{\prime} J_{\nu}(k a), \\
q_{n \nu} J_{\nu}\left(k_{0} a\right)+B_{\nu} H_{\nu}{ }^{(1)}\left(k_{0} a\right) & =B_{\nu}{ }^{\prime} J_{\nu}(k a), \\
p_{n \nu} k_{0} J_{\nu}{ }^{\prime}\left(k_{0} a\right)+A_{\nu} k_{0} H_{\nu}{ }^{(1)}{ }^{\prime}\left(k_{0} a\right)= & A_{\nu}{ }^{\prime} M k J_{\nu}{ }^{\prime}(k a) \\
& +B_{\nu}{ }^{\prime} i K(\nu / a) J_{\nu}(k a), \\
q_{n \nu} k_{0} J_{\nu}{ }^{\prime}\left(k_{0} a\right)+B_{\nu} k_{0} H_{\nu}{ }^{(1) \prime}\left(k_{0} a\right)= & -A_{\nu}{ }^{\prime} i K(\nu / a) J_{\nu}(k a) \\
& +B_{\nu}{ }^{\prime} M k J_{\nu}{ }^{\prime}(k a) .
\end{aligned}
$$

The determination of the coefficients $A_{\nu}, B_{\nu}$ from these equations is a routine matter. To simplify the final expressions it is well to introduce the following abbreviations

$$
\left.\begin{array}{c}
N_{H}=\frac{H_{\nu}^{(1) \prime}\left(k_{0} a\right)}{H_{\nu}{ }^{(1)}\left(k_{0} a\right)}-\left(M k / k_{0}\right) \frac{J_{\nu}{ }^{\prime}(k a)}{J_{\nu}(k a)}, \\
N_{J}=\frac{J_{\nu}{ }^{\prime}\left(k_{0} a\right)}{J_{\nu}\left(k_{0} a\right)}-\left(M k / k_{0}\right) \frac{J_{\nu}{ }^{\prime}(k a)}{J_{\nu}(k a)} .
\end{array}\right\}
$$

Because of well-known properties of cylindric functions, the difference is then,

$$
N_{H}-N_{J}=\left(2 i / \pi k_{0} a\right)\left[J_{\nu}\left(k_{0} a\right) H_{\nu}^{(1)}\left(k_{0} a\right)\right]^{-1} .
$$

Further, we designate

$$
\begin{aligned}
& P_{\nu}=\frac{N_{H} N_{J}-K^{2} \nu^{2} / k_{0}{ }^{2} a^{2}}{N_{H}{ }^{2}-K^{2} \nu^{2} / k_{0}{ }^{2} a^{2}} \cdot \frac{J_{\nu}\left(k_{0} a\right)}{H_{\nu}{ }^{(1)}\left(k_{0} a\right)}, \\
& Q_{\nu}=\left(2 K \nu / \pi k_{0}{ }^{2} a^{2}\right)-\frac{1}{N_{H}{ }^{2}-K^{2} \nu^{2} / k_{0}{ }^{2} a^{2}} \cdot \frac{1}{\left[H_{\nu}{ }^{(1)}\right]^{2}} .
\end{aligned}
$$

The expressions of the coefficients take then the form

$$
A_{\nu}=-p_{n \nu} P_{\nu}-q_{n \nu} Q_{\nu}, \quad B_{\nu}=p_{n \nu} Q_{\nu}-q_{n \nu} P_{\nu} .
$$

\section{APPROXIMATIONS FOR THIN POSTS}

According to our assumption, the argument $x=k_{0} a$ is a small number, therefore, the approximate expressions for $J_{\text {. }}(x)$ and $H_{\nu}{ }^{(1)}(x)$ are, for $\nu \geqslant 1$,

$$
\begin{aligned}
J_{\nu}(x) & =(1 / \nu !)(x / 2)^{\nu}, \\
H_{\nu}^{(1)}(x) & =-(i / \pi)(\nu-1) !(x / 2)^{-\nu} .
\end{aligned}
$$

The factors depending on $k_{0} a$ in the expressions (4.13) become, also for $\nu \geqslant 1$,

$$
\begin{aligned}
J_{\nu}(x) / H_{\nu}{ }^{(1)}(x) & =(i \pi / \nu !(\nu-1) !)(x / 2)^{2 \nu}, \\
{\left[H_{\nu}{ }^{(1)}(x)\right]^{-2} } & =-\left(\pi^{2} /(\nu-1) !(\nu-1) !\right)(x / 2)^{2 \nu} .
\end{aligned}
$$

The remaining factors in (4.13) have a particularly simple expression when not only $k_{0} a \ll<1$, but also $k a \ll 1$. Indeed, in this case,

$$
N_{H}=-(1+M)_{\nu} / k_{0} a, \quad N_{J}=(1-M) \nu / k_{0} a,
$$


and this leads to $(\nu \geqslant 1)$,

$$
\begin{aligned}
& P_{\nu}=i \frac{M^{2}-K^{2}-1}{(M+1)^{2}-K^{2}} \frac{\pi}{\nu !(\nu-1) !}\left(\frac{k_{0} a}{2}\right)^{2 \nu}, \\
& Q_{\nu}=-\frac{2 K}{(M+1)^{2}-K^{2}} \frac{\pi}{\nu !(\nu-1) !}\left(\frac{k_{0} a}{2}\right)^{2 \nu} .
\end{aligned}
$$

The case $\nu=0$ must be evaluated separately because, for small $x$,

$$
H_{0}^{(1)}(x)=1-(2 i / \pi) \ln \left(\frac{2}{\gamma x}\right) .
$$

The result is

$$
\begin{aligned}
& P_{0}=-i \pi(\epsilon-1)\left(k_{0} a / 2\right)^{2}\left[1-\frac{1}{2} \epsilon k_{0}{ }^{2} a^{2} \ln \left(\frac{1}{2} \gamma k_{0} a\right)\right], \\
& Q_{0}=0 .
\end{aligned}
$$

Thus, the coefficients $A_{0}, B_{0}, A_{1}, B_{1}$ are of the same order of magnitude containing the small factor $\left(k_{0} a\right)^{2}$. The convergence is rapid and the coefficients $A_{2}, B_{2}$ are already of the order $\left(k_{0} a\right)^{4}$.

Of course, the assumption $k a \ll 1$ imposes too great a limitation on the radius $a$ of the post because in ferrites the wave number $k$ is apt to be fairly large. However, for $k a$ not small, the magnitude of the expressions (4.13) is determined essentially by the factors (5.2) with $x=k_{0} a$. Indeed, it was ascertained by numerical calculation that the remaining factor (for not small $k a$ ) is not greatly dependent in magnitude on the index $\nu$ and remains of the same order for all $\nu$ larger than 0 . Thus, in this case also the convergence is very rapid and it is permissible to retain only the terms with $\nu=0$ and $\nu=1$.

\section{METHOD OF GREEN'S FUNCTION}

Having found the scattered wave of the first order, we proceed now to the next step in the program outlined in Sec. 3, namely, to the determination of the mirrored wave of the first order. We recall that the sum of the two waves, $E_{p 1}=E_{s 1}+E_{m 1}$ (or omitting the subscript $1, E_{p}=E_{s}+E_{m}$ ) has the following properties: it satisfies the wave equation (2.5) and it vanishes at the side walls of the wave guide, $y=0, y=\pi$. A convenient and well known method of finding a function of these properties is that of Green's function. A detailed explanation of it adapted to the case of the rectangular wave guide can be found in literature. ${ }^{10}$ Let Green's function be denoted by $G\left(x, y ; x^{\prime}, y^{\prime}\right)$. It is defined as satisfying the equation

$$
\begin{aligned}
\left(\frac{\partial^{2}}{\partial x^{2}}+\frac{\partial^{2}}{\partial y^{2}}+k_{0}^{2}\right) G\left(x, y ; x^{\prime}, y^{\prime}\right) & \\
= & -\delta\left(x-x^{\prime}\right) \delta\left(y-y^{\prime}\right)
\end{aligned}
$$

where the symbol $\delta(x)$ denotes Dirac's delta function. This means that Green's function $G$ has a logarithmic

${ }^{10}$ Compare, for instance, $\mathrm{Ph}$. M. Morse and H. Feshbach, Methods of Theoretical Physics (McGraw-Hill Book Company, Inc., New York, 1953), Part I, p. 804. singularity at the point $x=x^{\prime}, y=y^{\prime}$ and satisfies the homogeneous equation (2.5) at all other points. Moreover, it is subject to the boundary conditions

$$
G=0, \text { for } \quad \begin{gathered}
y=0, \quad y=\pi, \\
y^{\prime}=0, \quad y^{\prime}=\pi .
\end{gathered}
$$

The explicit expression of such a function is ${ }^{10}$

$$
\begin{gathered}
G\left(x, y ; x^{\prime}, y^{\prime}\right)=\sum_{n=1}^{\infty} G_{n} \\
G_{n}=\underset{\pi}{i} \gamma_{n}^{-1} \sin n y \sin n y^{\prime} \exp i \gamma_{n}\left|x-x^{\prime}\right|,
\end{gathered}
$$

where $\gamma_{n}$ is the parameter defined by the Eq. (2.14). In the special case $n>k_{0}$, the parameter $\gamma_{n}$ must be taken as positive imaginary.

The application of Green's function to our problem is as follows. Let us consider a region bounded by two curves $L_{1}$ and $L_{2}$ dotted in Fig. 2, in whose every point the wave equation (2.5) is valid. At a point $x=x^{\prime}, y=y^{\prime}$ of this region, marked in the figure by $P^{\prime}$, the wave potential $E_{p}$ of the required properties is represented by

$$
\begin{aligned}
E_{p}\left(x^{\prime}, y^{\prime}\right)=\int_{L}\left[G\left(x, y ; x^{\prime}, y^{\prime}\right) \frac{\partial E_{p}(x, y)}{\partial N}\right. & \\
& \left.-\frac{\partial G\left(x, y ; x^{\prime}, y^{\prime}\right)}{\partial N} E_{p}(x, y)\right] d l .
\end{aligned}
$$

The integral is extended over the two bounding lines, $L_{1}, L_{2}$, of the region, while $N$ denotes the outer normal at these lines. It is proved in the general theory that this integral is zero if taken over any closed curve surrounding both the singular point of $G$ (i.e., $P^{\prime}$ ) and the source of the radiation. Because the Green's function satisfies the conditions (6.2) the ferrite post may be regarded as the sole source of perturbation; therefore, the part of the integral (6.4) over the outer curve $L_{2}$ vanishes and only that over the inner boundary $L_{1}$ needs to be taken into account. In the integrand of (6.4) $E_{p}(x, y)$ is replaced by $E_{61}(x, y)$; this means that the effect of the waves $E_{s 2}(x, y), E_{s 3}(x, y)$, etc., is neglected. This is, however, exactly the degree of approximation which we wish to maintain according to the discussion of Sec. 2.

We take the path of integration infinitely close to the surface $r=a$ of the ferrite post, whence,

$$
\frac{\partial}{\partial N}=-\frac{\partial}{\partial r}, \quad d l=a d \varphi,
$$

we also use the simplified writing $E_{p}, E_{s}$ instead of 
$E_{p 1}, E_{z 1}$. Then we find

$$
\begin{aligned}
E_{p}\left(x^{\prime}, y^{\prime}\right)=-a \int_{0}^{2 \pi} & {\left[G\left(x, y ; x^{\prime}, y^{\prime}\right) \frac{\partial E_{s}(x, y)}{\partial r}\right.} \\
& \left.-\frac{\partial G\left(x, y ; x^{\prime}, y^{\prime}\right)}{\partial r} E_{s}(x, y)\right] d \varphi .
\end{aligned}
$$

\section{THE PERTURBATION WAVES}

Comparing the individual terms $G_{n}$ of Green's function (6.3) with the incident wave $E_{n}$ of Eq. (2.13), we find for $x>x^{\prime}$,

$$
G_{n}=(i / \pi) \gamma_{n}{ }^{-1} \sin n y^{\prime} \exp \left(-i n x^{\prime}\right) E_{n}(x, y) .
$$

Hence, the expansion of $G_{n}$ in polar coordinates defined by Eqs. (4.3) is reduced to the expansion (4.4) of $E_{n}$. The case $x<x^{\prime}$, is different only in that $x=r \cos \varphi$ and $x^{\prime}$ must be replaced by $-x$ and $-x^{\prime}$, respectively. This means that, in the expansion, $\varphi$ must be replaced by $(\pi-\varphi)$. Designating the summation variable by $s$, we obtain the general formula

$$
\begin{aligned}
G=(i / \pi) \sum_{s=1}^{\infty} \gamma_{s}^{-1} \sin s y^{\prime} \exp \left(\mp i s x^{\prime}\right) \\
\quad \times \sum_{\nu=0}^{\infty}( \pm 1)^{\nu} J_{\nu}\left(k_{0} r\right)\left[p_{s \nu} \cos \nu \varphi \pm q_{s \nu} \sin \nu \varphi\right]
\end{aligned}
$$

where the upper sign refers to the case $x^{\prime}<x$ and the lower to $x^{\prime}>x$. Since the expansion of the function $E_{8}$ is given by the series (4.7), the evaluation of the integral (6.6) does not present any difficulties and leads to the result

$$
\begin{aligned}
E_{p}\left(x^{\prime}, y^{\prime}\right)= & (2 / \pi) \sum_{s=1}^{\infty} \gamma_{s}^{-1} \sin s y^{\prime} \exp \left(\mp i \gamma_{s} x^{\prime}\right) \\
& \times\left[2 A_{0} p_{s 0}+\sum_{\nu=1}^{\infty}( \pm 1)^{\nu}\left(A_{\nu} p_{s \nu} \pm B_{\nu} q_{s \nu}\right)\right]
\end{aligned}
$$

or substituting the expressions (4.14) for $A_{v}, B_{v}$

$$
\begin{aligned}
E_{p}\left(x^{\prime}, y^{\prime}\right)=(-2 / \pi) \sum_{s=1}^{\infty} \gamma_{s}^{-1} \sin y^{\prime} \exp \left(\mp i \gamma_{s} x^{\prime}\right) \\
\times\left\{2 p_{n 0} p_{s 0} P_{0}+\sum_{\nu=1}^{\infty}( \pm 1) \nu\left[\left(p_{n \nu} p_{s \nu} \pm q_{n \nu}, q_{s v}\right) P_{\nu}\right.\right. \\
\left.\left.+\left(q_{n \nu} p_{s \nu} \mp p_{n \nu} q_{s v}\right) Q_{\nu}\right]\right\} .
\end{aligned}
$$

This formula represents the perturbation wave as a superposition of the characteristic modes of the empty wave guide. The upper sign indicates modes progressing in the negative $x^{\prime}$ direction. Since the coordinate $x$ describes the points of the post surface, the condition $x^{\prime}<x$ means that the solution with the upper sign is valid for points in Fig. 2 to the left of the whole surface of the post, i.e., $x^{\prime}<-a$. Therefore it may be called the

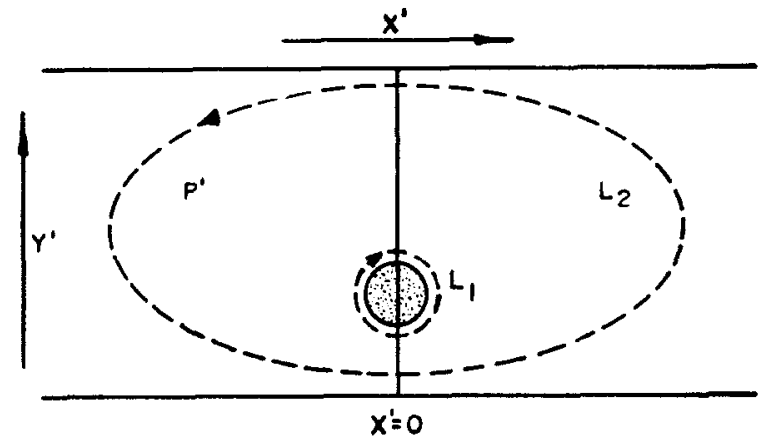

FIG. 2. Region of integration for Eq. (6.4).

reflected perturbation wave. On the other hand, the lower sign solution is conditional upon the inequalities $x^{\prime}>x$ or $x^{\prime}>a$, and at the same time it consists of modes progressing in the positive $x$-direction. Therefore, it will be designated as the transmitted periurbation wave.

It is well to point out that the solution (7.3) does not apply to the region at the surface of the ferrite post itself where $-a<x^{\prime}<a$. An evaluation of the perturbation in this region by means of our method would be rather cumbersome and the great advantage of simplicity offered by the procedure would be lost.

\section{CASE MOST IMPORTANT IN PRACTICE}

The conditions become particularly clean-cut when the incident wave (2.13) is selected so that it represents the fundamental mode, $n=1$, namely,

$$
E_{1}=\sin y \exp i \gamma_{1} x, \quad \gamma_{1}=\left(k_{0}^{2}-1\right)^{\frac{1}{2}},
$$

and when moreover the frequency is adjusted so as to make

$$
1<k_{0}<2
$$

This makes all the modes in the perturbation waves (7.3), from $s=2$ on, evanescent because already $\gamma_{2}=\left(k_{0}^{2}-4\right)^{\frac{1}{3}}$ is imaginary. At a sufficient distance from the post the strongly damped evanescent modes die out so that both in the reflected and the transmitted waves only the fundamental mode $s=1$ remains. Thus the sum over $s$ in the expression (7.3) is reduced to the single term $s=1$.

In addition, it must be remembered that we restrict our theory to the case when the ferrite post is thin and the convergence of the series (4.7) rapid. Under these circumstances, only terms in the sum (7.3) with $\nu=0$ and $\nu=1$ need to be taken into consideration. Therefore, at sufficiently large distances from the post the perturbation field takes a comparatively simple form.

The reflected wave is, for large negative $x^{\prime}$, given by,

$$
\begin{aligned}
E_{p}{ }^{(R)} & =C_{R} \sin y^{\prime} \exp \left(-i \gamma_{1} x^{\prime}\right), \\
C_{R} & =-\gamma_{1}^{-1}(2 / \pi)\left[2 p_{10}^{2} P_{0}+\left(p_{11}{ }^{2}+q_{11}{ }^{2}\right) P_{1}\right] .
\end{aligned}
$$


The transmitted wave, for large positive $x^{\prime}$,

$$
\begin{aligned}
E_{p}{ }^{(T)}=C_{T} \quad \sin y^{\prime} & \exp \left(i \gamma_{1} x^{\prime}\right), \\
C_{T}=-\gamma_{1}^{-1}(2 / \pi) & {\left[2 p_{10}{ }^{2} P_{0}\right.} \\
& \left.-\left(p_{11}{ }^{2}-q_{11}^{2}\right) P_{1}-2 p_{11} q_{11} Q_{1}\right] .
\end{aligned}
$$

In this case, the mode (8.5) is the same as that of the incident radiation, so that the transmitted wave serves only to modify the amplitude and phase of the incident mode, the total coefficient being $1+C_{T}$.

The results obtained for ferrites, of course, can be specialized for the simpler case of dielectric posts with a scalar permeability $\mu$ by letting $\kappa=K=0$. In this case $Q_{\nu}$ vanishes for all subscripts $\nu$ and for all diameters of the post. Further, if the post is nonmagnetic, $(\mu=1)$, $P_{1}$ becomes negligible for very small radii $a$, as is evidenced by the Eqs. (5.4). Hence,

$$
C_{R}=C_{T}=-(4 / \pi) \alpha^{-1} p_{01}^{2} P_{0} .
$$

This agrees, in fact, with the expression given by Marcuvitz ${ }^{8}$ for the very thin nonmagnetic dielectric post.

\section{REMARK ON THE EVANESCENT MODES}

The evanescent modes do not play any role in the energy balance because they do not transport any energy. This is due to a property which is apparent from the expressions (2.13) and (2.4) for the electric and magnetic vectors. When $\gamma_{n}=i\left|\gamma_{n}\right|$, as is the case in evanescent modes, the electric vector in our representation is real and the magnetic imaginary. This means that the two components have a phase difference of $(\pi / 2)$ so that Poynting's vector vanishes.

Nevertheless, the evanescent modes are not entirely without interest since they contribute to the amplitude of the field, though not to its energy. It is, therefore, pertinent to ask about their intensity at comparatively small distances from the ferrite post where they are not negligible. In particular, it is important to ascertain that the series (7.3) are convergent when the evanescent modes are taken into consideration. Fortunately, the convergence is beyond doubt. Indeed, for large values of $s$ there holds the approximate relation $\gamma_{s} \simeq i s$, whence

$$
\epsilon_{s} \stackrel{\pi}{2}+i \underset{k_{0}}{2}
$$

and

$$
\cos \nu \epsilon_{s} \simeq \sin \nu \epsilon_{s} \simeq \frac{1}{2}\left(-2 i s / k_{0}\right)^{\nu} .
$$

Taking into account the expressions (4.5) and (5.4) we see that in the high order terms of the double expansion (7.3) the decisive factors on which the convergence depends are $s\left(k_{0} a\right)^{2 \nu} \exp \left(-s\left|x^{\prime}\right|\right) / \nu !(\nu-1) !$, which ensure the convergence of the sum with respect to $\nu$ as well as of that with respect to $s$.

At the same time we see that the evaluation of the total field at small distances from the ferrite post would be extremely cumbersome, even apart from the fact, mentioned in Sec. 7, that our expressions do not apply to the region $-a<x^{\prime}<a$. This observation gives added force to the conclusion that our method is suitable only for the treatment of thin posts where higher order approximations are not required.

Nevertheless, it is in order to ask how an evanescent incident wave is diffracted by a ferrite post. Considering that the evanescent wave does not carry any energy, it may be argued on physical grounds that the diffraction radiation must be also energy-free and must consist of cylindrical evanescent waves. More generally, the conclusion seems justified that in all its subsequent transformations by reflection and scattering the evanescent wave remains outside the energy balance. Thus, in estimating the intensities of the successive scattered waves of Sec. 3, only the normal modes need to be considered. The formulas (5.4) referring to thin posts show that in this case every successive scattering reduces the intensity in the ratio $\left(k_{0} a / 2\right)^{4}$. It appears, therefore, that the convergence of the successive approximations is considerably better than indicated by the factor $(a / d)$ alone. Indeed, it seems probable that the approximation calculated in the final formulas of Secs. 7 and 8 is adequate even when only the wavelength $\lambda$ is large compared with the radius $a$ of the post, but the distance $d$ to the nearest wall is not large. However, in view of the qualitative nature of this argument and of the absence of an analytical field representation for the region $-a<x^{\prime}<a$, it is perhaps safer to retain the assumption $d \gg a$.

\section{RESONANCE OSCILLATIONS IN A CAVITY CONTAINING A FERRITE POST}

The conclusions of the preceding section may be conveniently tested by observing the effect of the ferrite post on the characteristics of a rectangular cavity. Let the cavity be produced by two shorting plates, $S_{1}$ and $S_{2}$ in the rectangular wave guide containing the post described in Sec. 2. Let them be placed in the respective positions $x=-L_{1}$ and $x=L_{2}$ at considerable distances from the axis of the post. (For brevity we write in this section $x, y$ instead of $x^{\prime}, y^{\prime}$.) We restrict the analysis to the case, discussed in the preceding section, when the condition (8.2) obtains, so that at sufficient distances from the post the field consists of two fundamental modes progressing in opposite directions. In the two halves (positive and negative) of the cavity the waves running toward the post can then be considered as the incident waves which shall be denoted by $A_{1} \sin y \exp \left(i \gamma_{1} x\right)$ and $A_{2} \sin y \exp \left(-i \gamma_{1} x\right)$, respectively, and shall be referred to as the first and the second incident wave. Thus, the total electric field in the neighborhood of $S_{1}$ is expressed by

$$
\begin{aligned}
& E=\left\{A_{1} \exp \left(i \gamma_{1} x\right)+\left[A_{1} C_{R}+A_{2}\left(1+C_{T}{ }^{\prime}\right)\right]\right. \\
&\left.\times \exp \left(-i \gamma_{1} x\right)\right\} \sin y .
\end{aligned}
$$

The terms with $A_{1} C_{R}$ and $A_{2} C_{T^{\prime}}$, respectively, arise from the reflection of the first incident wave and from the transmission of the second. At the shorting plate $S_{1}$ the boundary condition (2.2) requires the vanishing of 
$E$ for $x=-L_{1}$, whence,

$$
A_{1}\left[\exp \left(-2 i \gamma_{1} L_{1}\right)+C_{R}\right]+A_{2}\left(1+C_{T^{\prime}}\right)=0 .
$$

A similar expression of the field and boundary conditions obtains at the shorting plate $S_{2}$, the latter being,

$$
A_{1}\left(1+C_{T}\right)+A_{2}\left[\exp \left(-2 i \gamma_{1} L_{2}\right)+C_{R}{ }^{\prime}\right]=0 .
$$

The coefficients $C_{R}{ }^{\prime}, C_{T}{ }^{\prime}$ of the reflection and transmission of the second incident wave are, in general, not the same as $C_{R}, C_{T}$ of the first, because from the point of view of the second wave the position of the post is different, so that

$$
C_{R}{ }^{\prime}(d)=C_{R}(\pi-d), \quad C_{T}{ }^{\prime}(d)=C_{T}(\pi-d) .
$$

The compatibility condition of the two boundary equations (10.2) and (10.3) is

$$
\begin{aligned}
{\left[C_{R}+\exp \left(-2 i \gamma_{1} L_{1}\right)\right] \cdot } & {\left[C_{R}{ }^{\prime}+\exp \left(-2 i \gamma_{1} L_{2}\right)\right] } \\
& -\left(1+C_{T}\right)\left(1+C_{T^{\prime}}\right)=0 .
\end{aligned}
$$

Inasmuch as the coefficients $C_{R}, C_{T}, C_{R}{ }^{\prime}, C_{T}{ }^{\prime}$, and the wave number $\gamma_{1}$, are known functions of the frequency $\omega, \mathrm{Eq}$. (10.5) represents the condition fixing the resonance frequency of the cavity. It can be used to determine the resonance frequency when the dimensions of the cavity are given. What we propose to do is to compare a cavity with a ferrite post (propagation constant $\gamma_{1}$, frequency $\omega$ ) with a cavity of the same size without the post (propagation constant $\gamma_{10}$, frequency $\omega_{0}$ ) so that

$$
\gamma_{1}-\gamma_{10}=\Delta \gamma_{1} ; \quad \gamma_{10}=\gamma_{1}-\Delta \gamma_{1}
$$

In the absence of a ferrite post the coefficients $C_{R}$,
$C_{T}$ vanish, and Eq. (10.5) is reduced to

$$
\exp \left[-2 i\left(\gamma_{1}-\Delta \gamma_{1}\right)\left(L_{1}+L_{2}\right)\right]=1,
$$

or for the fundamental eigenmode,

$$
\gamma_{1}\left(L_{1}+L_{2}\right)=\pi+\left(L_{1}+L_{2}\right) \Delta \gamma_{1}
$$

For thin posts the coefficients $C$ are small numbers whose squares and products are negligible. Hence, in Eq. (10.5) only linear terms in $C$ and $\Delta \gamma_{1}$, should be retained. There follows,

$$
\begin{array}{r}
\left(L_{1}+L_{2}\right) \Delta \gamma_{1}=(i / 2)\left[C_{T}+C_{T}^{\prime}-C_{R} \exp \left(-2 i \gamma_{1} L_{2}\right)\right. \\
\left.-C_{R^{\prime}} \exp \left(-2 i \gamma_{1} L_{1}\right)\right] .
\end{array}
$$

This relation may become useful for measuring certain combinations of the ferrite constants. Indeed, since each of the three functions $P_{0}, P_{1}, Q_{1}$ defined by Eqs. (5.4) depends on the constants in a different way, and since the weight with which these functions enter into the coefficients, $C$, changes with the parameter $d$, it should be possible to determine all three constants $\epsilon$, $\mu_{1}, \kappa$ by a series of measurements with different positions of the post. It is true that for different positions of the post the resonance frequency will not be quite the same and, therefore, the constants will also slightly change since they are (unknown) functions of the frequency. However, in all ranges not too close to the absorption frequency of the ferrite, the change of the constants in a narrow spectral interval is negligible because it is of the same order of magnitude as that of the already neglected quadratic terms in the coefficients.

A summary of the results is given in the introduction. We wish to express our thanks to Dr. B. A. Lengyel for his interest and constructive criticism.

\title{
Quantitative Study of Substructure Characteristics and Correlation to Tensile-Property of Nickel and Nickel Alloy
}

\author{
Sigmund WeissmanN \\ College of Engineering, Rutgers University, New Brunswick, New Jersey
}

(Received April 27, 1956)

\begin{abstract}
The application of a method combining $x$-ray microscopy and diffraction analysis to the study of the substructure of nickel and nickel alloys disclosed the coexistence of three orders of magnitude of substructural entities. These three orders are structurally interrelated and distinguished by different ranges of size, disorientation angle, and lattice misalignment. The statistical analysis of the data disclosed that for the two largest orders of substructural entities increasing amounts of room temperature deformation cause (1) a decrease in the mean size and a decrease in the spread of the size distribution, (2) an increase in the mean disorientation angle between substructural entities of the same order and an increase in the spread of the distribution of angles, and (3) an increase in the mean lattice misalignment and spread in the distribution of misalignment. Progressive room temperature deformation causes the low-angle boundaries to become increasingly unsymmetrical and greatly increases the number of the smallest substructural entities. The rise of flow stress as a function of room temperature deformation was correlated to the systematic modification of substructure characteristics.
\end{abstract}

\section{INTRODUCTION}

$\mathrm{C}$ ONSIDERABLE interest has been focussed during the past years on the relationship between the substructure formation resulting from the deformation of metals and the strength properties which the metals exhibit. Quantitative investigations relating substructure to the tensile properties of nickel and nickel alloys have shown that increasing amounts of room tempera- 